\title{
The role of serum C-reactive protein in the diagnosis of periprosthetic shoulder infection
}

\author{
Doruk Akgün ${ }^{1} \cdot$ Mats Wiethölter $^{1} \cdot$ Paul Siegert $^{1} \cdot$ Victor Danzinger $^{1} \cdot$ Marvin Minkus $^{1} \cdot$ Karl Friedrich Braun $^{1}$. \\ Philipp Moroder ${ }^{1}$
}

Received: 16 October 2020 / Accepted: 6 January 2021 / Published online: 30 January 2021

(c) The Author(s) 2021

\begin{abstract}
Introduction There is a paucity of literature regarding serum C-reactive protein (CRP) in the evaluation of a shoulder periprosthetic joint infection (PJI). The purpose of the current study was to establish cutoff values for diagnosing shoulder PJI and evaluate the influence of the type of infecting microorganism and the classification subgroups according to last proposed International Consensus Meeting (ICM) criteria on the CRP level.

Materials and methods A retrospective analysis of all 136 patients, who underwent septic or aseptic revision shoulder arthroplasty in our institution between January 2010 and December 2019, was performed. Shoulder PJI was defined according to the last proposed definition criteria of the ICM. Serum CRP levels were compared between infected and non-infected cases, between infection subgroups, as well as between different species of infecting microorganisms. A receiver-operating characteristic (ROC) analysis was performed to display sensitivity and specificity of serum CRP level for shoulder PJI.

Results A total of 52 patients (38\%) were classified as infected, 18 meeting the criteria for definitive infection, 26 for probable infection and 8 for possible infection. According to the ROC curve, an optimized serum CRP threshold of $7.2 \mathrm{mg} / \mathrm{l} \mathrm{had}$ a sensitivity of $69 \%$ and specificity of $74 \%$ (area under curve $=0.72$ ). Patients with definitive infection group demonstrated significantly higher median serum CRP levels $(24.3 \mathrm{mg} / \mathrm{l})$, when compared to probable, possible infection groups and PJI unlikely group $(8 \mathrm{mg} / 1,8.3 \mathrm{mg} / 1,3.6 \mathrm{mg} / \mathrm{l}$, respectively, $p<0.05)$. The most common isolated microorganism was Cutibacterium acnes in 25 patients (48\%) followed by coagulase-negative staphylococci (CNS) in 20 patients (39\%). Patients with a PJI caused by high-virulent microorganisms had a significantly higher median serum CRP level compared to patients with PJI caused by low-virulent microorganisms ( $48 \mathrm{mg} / \mathrm{l} \mathrm{vs.} 11.3 \mathrm{mg} / \mathrm{l}, p=0.04)$.

Conclusions Serum CRP showed a low sensitivity and specificity for the diagnosis of shoulder PJI, even applying cutoffs optimized by receiver-operating curve analysis. Low-virulent microorganisms and patients with probable and possible infections are associated with lower CRP levels compared to patients with definitive infection and infections caused by high-virulent microorganisms.
\end{abstract}

Level of evidence Diagnostic Level III.

Keywords C-reactive protein $\cdot$ Periprosthetic shoulder infection $\cdot$ Definition $\cdot$ Low-virulence

\section{Introduction}

Doruk Akgün and Mats Wiethölter contributed equally to this work.

Doruk Akgün

doruk.akguen@charite.de

1 Department for Shoulder and Elbow Surgery, Center for Musculoskeletal Surgery, Humboldt-Universität zu Berlin, and Berlin Institute of Health, Charité-University Medicine Berlin, Corporate Member of Freie Universität Berlin, Augustenburger Platz 1, 13353 Berlin, Germany
The accurate diagnosis of shoulder periprosthetic joint infection (PJI) continues to pose a difficult task due to the subtle clinical presentation of common low-grade infections, especially involving Cutibacterium acnes, which is identified in both primary and revision shoulder arthroplasties in up to $70 \%$ of the cases with positive cultures [1-3]. Although acute infections are readily detectable mostly due to clinical symptoms and high levels of inflammatory biomarkers, chronic low-grade infections present a unique diagnostic and 
therapeutic challenge. C-reactive protein (CRP) has been shown to be a powerful parameter for detecting periprosthetic joint infections and recommended by the American Academy of Orthopaedic Surgeons (AAOS) [4] and the International Consensus Meeting (ICM) [5] as first-line test in the diagnosis of PJI. However, recent literature showed high false-negative rates, especially in patients with lowvirulent microorganisms [6-9].

There is a paucity of literature regarding serum CRP in the evaluation of shoulder PJI and existing studies show sensitivities varying between 30 and 91\% [10-14]. These differences in sensitivities between studies can be related to the different definitions criteria used in these studies and different types of bacteria. Recently, new criteria was proposed for shoulder PJI classifying it into 4 subgroups; (1) definitive infection, (2) probable infection, (3) possible infection and (4) infection unlikely [15]. This stratification allows for more homogenous groups with different characteristics of PJI and levels of inflammation. The purpose of the current study was to establish cutoff values for diagnosing shoulder PJI and evaluate the influence of the type of infecting microorganism and the classification subgroups on the CRP level.

\section{Materials and methods}

A retrospective analysis of all patients, who underwent septic or aseptic revision shoulder arthroplasty in our institution between January 2010 and December 2019 was performed. Following data were recorded for each patient: gender and age, involved joint, clinical symptoms, surgical history of the involved joint, type of arthroplasty, concurrent antibiotic treatment, as well as radiological assessment. The standard diagnostic protocol to identify PJI included the following assessments in all patients; Laboratory values including C-reactive protein, serum leucocyte count as well as results of preoperative aspiration, if performed, including leucocyte count, neutrophil percentage, microbiologic and histopathologic results, radiological and intraoperative evaluation of the component loosening and intraoperative findings, such as cloudy fluid or gross intra-articular purulence and microbiological and pathological results of revision surgery. Furthermore, the total number of intraoperative tissue samples and the incidence of positive cultures were recorded for each patient. Patients with less than two tissue specimens for culture were excluded. Sonication fluid cultures were incorporated into the infection criteria as microbiologic results used for the infection definition.

Specimens for microbiological analysis were collected with a new sterile instrument each time, were placed directly into sterile containers without touching by hand and sent immediately with retrieved implants to our microbiology laboratory for further analysis within $1 \mathrm{~h}$ after surgery. The microbiologic specimen as well as sonication fluid cultures were placed onto aerobic and anaerobic sheep blood agar plates and incubated for 14 days. Sonication was performed as previously described in every case after January 2014 [16]. Shoulder PJI was diagnosed according to the last proposed definition criteria of the ICM and based on this definition CRP levels of greater than $10 \mathrm{mg} / \mathrm{l}$ were considered elevated [15]. According to these criteria, patients were classified into 4 infection subgroups: (1) definitive infection, (2) probable infection, (3) possible infection and (4) infection unlikely. Meeting one of the following criteria was diagnostic of definitive periprosthetic shoulder infection: (1) a sinus tract communicating with the prosthesis; (2) gross intra-articular pus; (3) two positive cultures with phenotypically identical virulent organisms. In the lack of these defining signs, weighted minor criteria (Table 1) are summed and used to distinguish between probable, possible and unlikely infection.

The three categories in these less distinct scenarios are defined as follows:

- Six or greater with identified organism: probable infection.

- Six or greater without identified organism: possible infection.

- Fewer than six.

- Single positive culture virulent organism: possible infection.

- Two positive culture low-virulence organisms: possible infection.

- Negative cultures or only single positive culture for lowvirulent organism: infection unlikely [15, 17].

Table 1 Minor criteria

\begin{tabular}{ll}
\hline & Weight \\
\hline Unexpected wound drainage & 4 \\
Single positive tissue culture (virulent organism) & 3 \\
Single positive tissue culture (low-virulence organism) & 1 \\
Second positive tissue culture (identical low-virulence & 3 \\
$\quad$ organism) & 3 \\
Humeral loosening & 3 \\
Positive frozen section (5 PMN in at least 5 high-power & \\
$\quad$ fields) & 3 \\
Positive preoperative aspirate culture (low or high virulence) & 3 \\
Elevated synovial neutrophil percentage $(>80 \%)$ & 2 \\
Elevated synovial WBC $(>3000$ cells $/ \mu \mathrm{l})$ & 2 \\
Elevated ESR $(>30 \mathrm{~mm} / \mathrm{h})$ & 2 \\
Elevated CRP $(>10 \mathrm{mg} / \mathrm{l})$ & 2 \\
Cloudy fluid & 2 \\
\hline
\end{tabular}




\section{Statistical analysis}

Chi-squared and Fisher's exact tests were used to find significant differences between categorical variables. The twosample $t$ test (for parametric distribution) or Mann-Whitney $U$ test (for non-parametric distribution) was used to compare continuous variables between the groups. Median serum CRP levels were calculated for various species of microorganisms [S. aureus, C. acnes, coagulase-negative staphylococci (CNS)] and for definitive, probable, possible infection and infection unlikely groups. Groups were then compared to each other for a statistically significant difference using Kruskal-Wallis test adjusting for multiple comparisons with Dunn's test. When analyzing species-specific results, monomicrobial and polymicrobial infections with only the same species were compared. For a further analysis of the diagnostic utility of serum CRP in all patients, patients in the definitive, probable and possible infection groups were combined and defined as infection group and patients in the infection unlikely group were defined as noninfection group. A receiver-operating characteristic (ROC) analysis was performed to display sensitivity and specificity of serum CRP level for shoulder PJI. The area under the curve (AUC) was calculated and the optimum cutoff point was determined using the maximized Youden's index. The results were expressed as mean and standard deviation (SD) or as number and percentage. A $p$ value $<0.05$ was considered significant. SPSS version 20 (SPSS Inc., Chicago, Illinois) was used for the statistical analyses.

\section{Results}

One hundred and thirty-six patients were included in this study. The mean age and standard deviation (SD) of the patients were $70 \pm 10.3$ years (range 37-91) and 79 patients were females $(58 \%)$. A total of 10 patients $(7 \%)$ had undergone at least 1 previous septic revision, $24(18 \%)$ had undergone an aseptic revision and $102(75 \%)$ had developed a PJI after the initial arthroplasty. The median interval from last surgical treatment until the revision surgery at our institution was 18.6 months $(0.7-166)$. The type of arthroplasty at the time of revision surgery was hemiarthroplasty in 48 patients, total shoulder arthroplasty in 38 and reverse shoulder arthroplasty in 50 patients. Besides infection, the reasons for revision surgery included loosening of the components, overstuffing, secondary rotator cuff insufficiency and instability.

A total of 52 patients (38\%) were classified as infected, 18 meeting the criteria for definitive infection, 26 for probable infection and 8 for possible infection. In $84(62 \%)$ patients, the infection was considered unlikely. A total of 560 cultures were taken in all patients and $142(25 \%)$ were positive. A polymicrobial infection was identified in 13 patients (25\%). Further demographic and clinical characteristics of the patients are summarized in Table 2 .

There was a significant difference in serum CRP levels between infection and non-infection groups $(27 \mathrm{mg} / \mathrm{l} \mathrm{vs}$. $7.1 \mathrm{mg} / \mathrm{l}, p<0.001)$. A serum CRP threshold of $10 \mathrm{mg} / \mathrm{l} \mathrm{had}$ a sensitivity of $58 \%$ and a specificity of $80 \%$. According to the ROC curve, an optimized serum CRP threshold of $7.2 \mathrm{mg} / \mathrm{l}$ had a sensitivity of $69 \%$ and specificity of $74 \%$ $(\mathrm{AUC}=0.72)$ (Fig. 1). Twenty-two patients $(42.3 \%)$ from the infection group had a normal $(<10 \mathrm{mg} / \mathrm{l})$ serum CRP level prior to revision surgery. When a diagnostic threshold of $7.2 \mathrm{mg} / \mathrm{l}$ is used, 16 patients ( $31 \%$ ) had still a false-negative CRP result.

When grouping infected patients by infection subgroups, patients in the definitive infection group demonstrated significantly higher median serum CRP levels $(24.3 \mathrm{mg} / \mathrm{l})$, when compared to probable, possible infection groups and PJI unlikely group $(8 \mathrm{mg} / \mathrm{l}, 8.3 \mathrm{mg} / \mathrm{l}, 3.6 \mathrm{mg} / \mathrm{l}$, respectively, $p<0.05)$ (Fig. 2). While 16 of 26 patients $(62 \%)$ with probable infection, 5 of 8 patients $(63 \%)$ with possible infection and 71 of 84 patients (84\%) without shoulder PJI had a normal $(<10 \mathrm{mg} / \mathrm{l})$ serum CRP level, only 1 of 18 patients $(6 \%)$ with definitive infection had a normal CRP level. Interestingly, patients with normal preoperative CRP had similar rates of positive cultures compared to patients with elevated $\operatorname{CRP}(46 / 96(48 \%)$ vs. 78/137 (57\%), $p=0.2)$.

The most common isolated microorganism was Cutibacterium acnes in 25 patients (48\%) followed by CNS in 20 patients (39\%). Staphylococcus aureus was isolated in 6
Table 2 Patient demographics and CRP findings

\begin{tabular}{|c|c|c|c|c|}
\hline Variable & All patients, $n=136$ & $\begin{array}{l}\text { Infection group, } \\
n=52\end{array}$ & $\begin{array}{l}\text { Non-infection } \\
\text { group, } n=84\end{array}$ & $p$ value \\
\hline Mean age, year ${ }^{\mathrm{a}}$ & $70 \pm 10.3$ & $70.6 \pm 10$ & $69.6 \pm 10.4$ & 0.6 \\
\hline \multicolumn{5}{|l|}{ Gender $^{\mathrm{b}}$} \\
\hline Male & $57(42)$ & $27(52)$ & $30(36)$ & 0.08 \\
\hline Female & $79(58)$ & $25(48)$ & $54(64)$ & \\
\hline $\mathrm{CRP}$ at admission $(\mathrm{mg} / \mathrm{l})^{\mathrm{a}}$ & $14.7 \pm 27$ & $27 \pm 39$ & $7.1 \pm 11$ & 0.0001 \\
\hline
\end{tabular}

${ }^{\text {a }}$ The values are given as the mean and the standard deviation

${ }^{\mathrm{b}}$ The values are given as the number with the percentage of the group in parentheses 


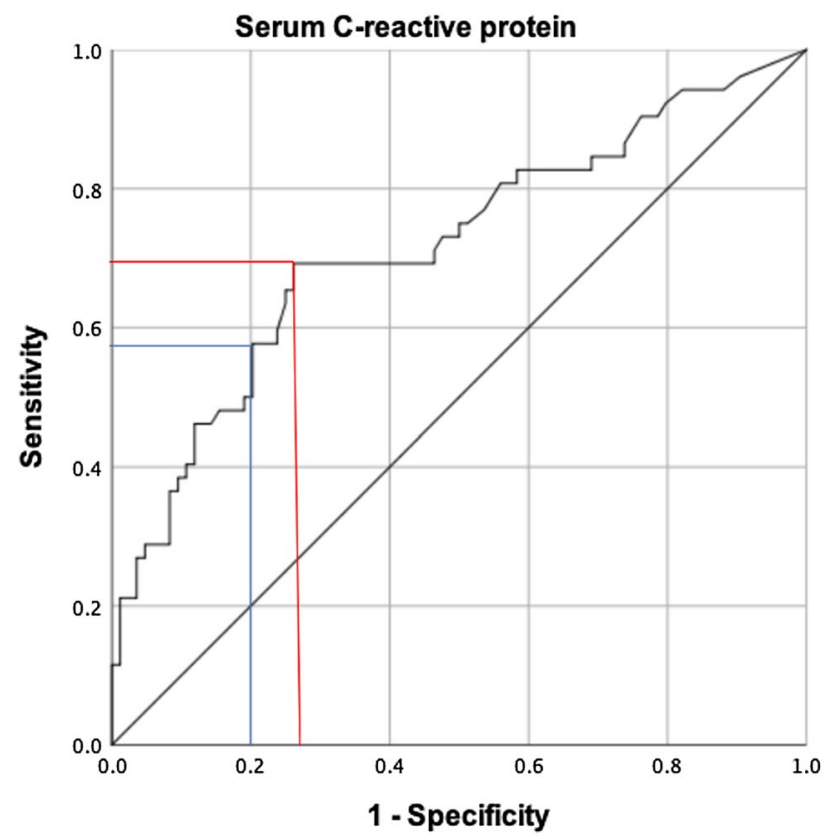

Fig. 1 Receiver-operating characteristics curve for serum C-reactive protein. Sensitivity is plotted against $100 \%$ specificity. The red and blue lines mark the sensitivity and 1-specificity for the optimized threshold (Youden's index) of $7.2 \mathrm{mg} / \mathrm{l}$ and ICM definition cutoff of $10 \mathrm{mg} / \mathrm{l}$, respectively

patients with definitive infection and in 2 patients with probable infection. Table 3 summarizes the causative pathogens. Patients with $C$. acnes and/or CNS infection represented $73 \%$ of all shoulder PJIs in patients with a normal CRP level. When grouping microorganisms by species, patients with $S$. aureus, CNS and $C$. acnes had a significantly higher median serum CRP levels (46 mg/l, $12.9 \mathrm{mg} / \mathrm{l}$ and $12.5 \mathrm{mg} / \mathrm{l}$, respectively) compared with patients with unlikely PJI $(3.6 \mathrm{mg} / \mathrm{l}$, $p<0.05)$. Although the patients with $\mathrm{S}$. aureus had a higher median serum CRP level compared to patients with CNS and $C$. acnes, this difference was not statistically significant (Fig. 3). Furthermore, patients with a PJI caused by highvirulent microorganisms had a higher median serum CRP level compared to patients with PJI caused by low-virulent microorganisms (48 mg/l vs. $11.3 \mathrm{mg} / \mathrm{l}, p=0.04$ ) (Fig. 4).

\section{Discussion}

Serum CRP has been an important biomarker for the diagnosis of periprosthetic infection for a long time. However, recent studies were able to show a high false-negative rate in patients with PJI, especially infected by low-virulent microorganisms [6-9]. Furthermore, there is still a paucity of literature regarding serum CRP in the evaluation of a shoulder PJI. In the last proposed ICM definition of shoulder PJI, an elevated serum CRP level was defined as a minor criteria and the threshold was determined as $10 \mathrm{mg} / \mathrm{l}$ according to the hip and knee PJI literature $[15,18]$. However, our study demonstrated a low sensitivity and specificity of $58 \%$ and $80 \%$, respectively, of serum CRP threshold of $10 \mathrm{mg} / \mathrm{l}$. Furthermore, we were able to find a more optimized threshold of $7.2 \mathrm{mg} / \mathrm{l}$ with a sensitivity of $69 \%$ and specificity of $74 \%$. While missing almost half of the patients with a shoulder PJI if a cutoff value of $10 \mathrm{mg} / \mathrm{l}$ is applied, only one-third of the patients had a false-negative serum CRP level prior to revision surgery, if a diagnostic threshold of $7.2 \mathrm{mg} / \mathrm{l}$ is used.

Piper et al. have investigated the role of serum CRP in hip, knee and shoulder PJI, as well as in patients with spinal implant infections [14]. They found out, that CRP showed the lowest sensitivity for the diagnosis of shoulder PJI, even applying cutoffs optimized using receiving operating curve
Fig. 2 Box-and-whisker plot showing dependency of serum C-reactive protein (CRP) level on the different periprosthetic joint infection (PJI) classification subgroups. The horizontal black line marks the median CRP level; the box, the interquartile range; and the whiskers, the minimum and maximum. Asterisks indicate groups with significantly higher median CRP levels compared to groups without asterisks

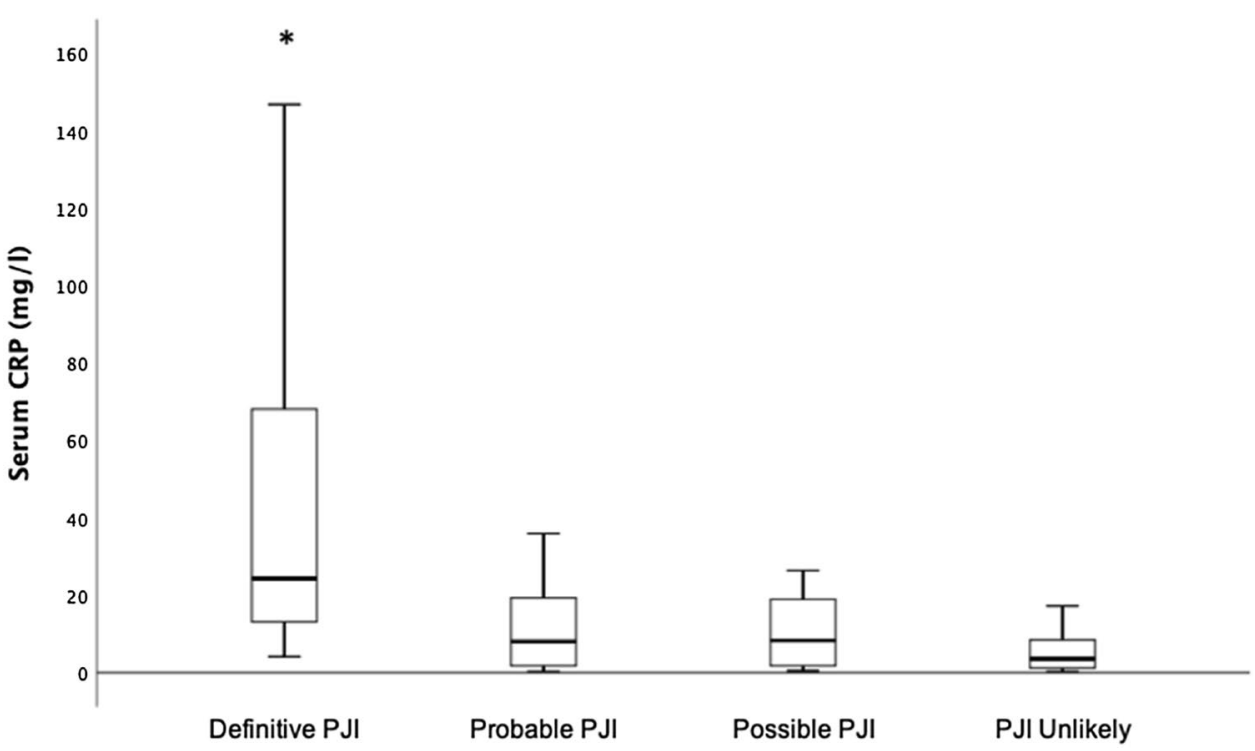


Table 3 Microorganisms identified in patients with PJI

\begin{tabular}{ll}
\hline Microorganisms & $\begin{array}{l}\text { No of patients } \\
\text { with PJI, } n=52 \\
(\%)\end{array}$ \\
\hline Cutibacterium acnes & $25(48)$ \\
Coagulase-negative staphylococci & $20(39)$ \\
Staphylococcus aureus & $8(15)$ \\
Enterococcus faecalis & $2(4)$ \\
Streptococcus spp. & $2(4)$ \\
Others & $4(8)$ \\
Polymicrobial & $13(25)$ \\
Negative microbiology & $6(12)$ \\
\hline
\end{tabular}

analysis. The optimized CRP cutoff was $7 \mathrm{mg} / \mathrm{l}$ with a sensitivity of $63 \%$ and specificity of $73 \%$, which is similar to the results of the current study. Other studies reported sensitivities of CRP varying between 30 and $91 \%$ [10-12]. In a recent meta-analysis, Nelson et al. reported, that $62.1 \%$ of patients, who were treated for shoulder PJI had an elevated CRP level [13].

The high prevalence of low-virulent microorganisms in CRP-negative PJIs observed in our study had previously been reported by the other authors [19-21]. In a study by McArthur, low-virulent microorganisms were significantly more often associated with normal CRP results compared
Fig. 3 Box-and-whisker plot showing dependency of serum C-reactive protein (CRP) level on the infecting microorganism and patients with unlikely periprosthetic joint infection (PJI). The horizontal black line marks the median CRP level; the box, the interquartile range; and the whiskers, the minimum and maximum. Asterisks indicate groups with significantly higher median CRP levels compared to groups without asterisks. Staphylococcus aureus, $n=5$; coagulasenegative staphylococci (CNS), $n=10$; Cutibacterium acnes, $n=14$; infection unlikely, $n=84$

Fig. 4 Box-and-whisker plot showing dependency of serum $\mathrm{C}$-reactive protein (CRP) level on the virulence of periprosthetic joint infection (PJI). The horizontal black line marks the median CRP level; the box, the interquartile range; and the whiskers, the minimum and maximum. Asterisks indicate groups with significantly higher median CRP levels compared to groups without asterisks. Highvirulent PJI, $n=10$; low-virulent PJI, $n=36$
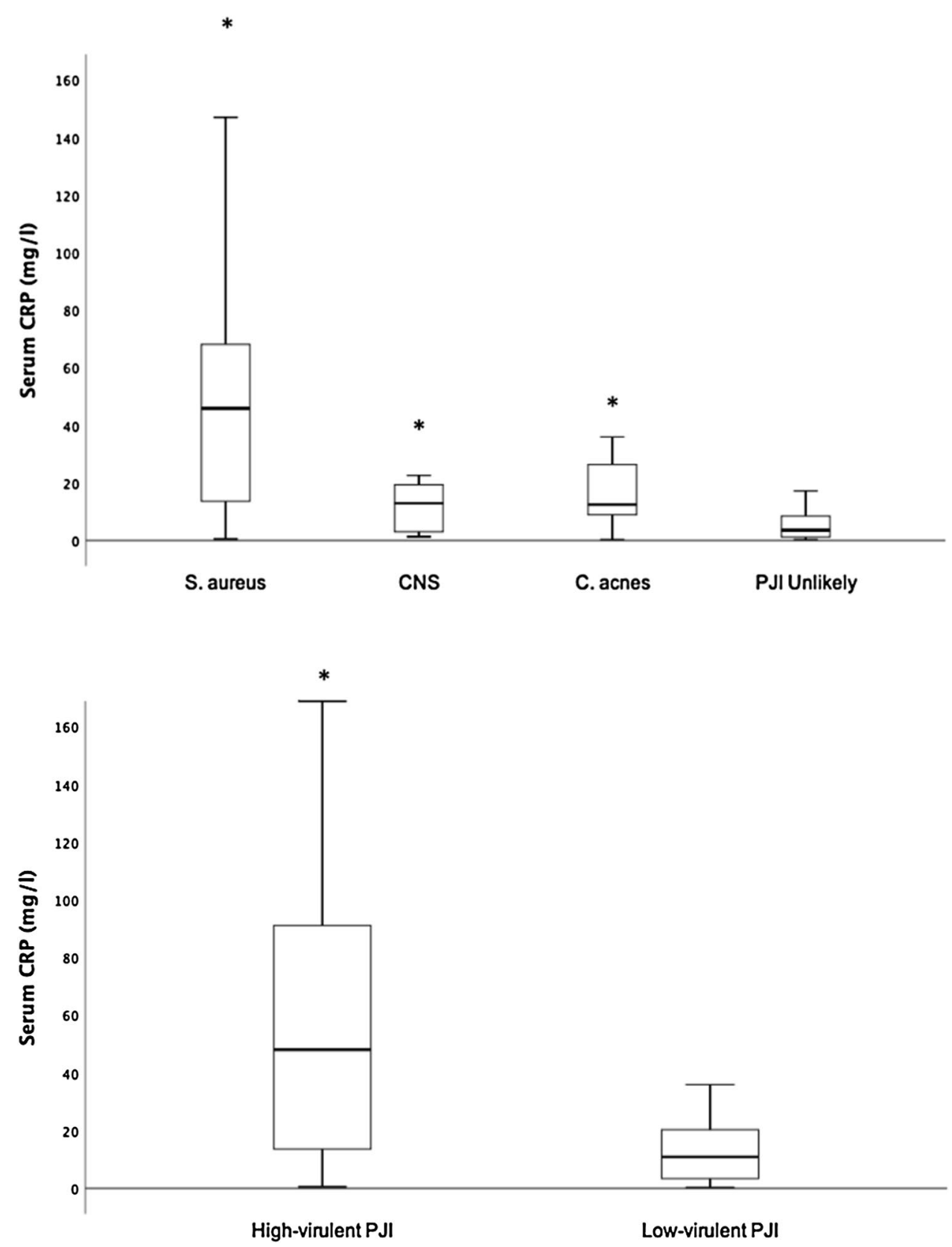
to high-virulent microorganisms. Obvious clinical signs of infection such as redness, swelling, sinus tract formation and fever are rarely encountered in infections caused by these low-virulent microorganisms and especially in these cases, CRP should not be used alone to rule out a diagnosis of shoulder PJI [22]. Our results also support the hypothesis that CRP response can be weak or even non-existent in patients with PJI caused by low-virulent microorganisms. This difference can be attributed to the fact that high-virulent microorganisms cause an acute planktonic infection inducing a high inflammation with release of cytokines and elevation of CRP, while low-virulent microorganisms adhere rapidly to implant surfaces forming biofilms, where they escape from the host immune system, which results in reduced inflammation $[6,23,24]$. This is especially true for $C$. acnes, which is a Gram-positive slow-growing microorganism. It can isolate itself from the host defense by forming a biofilm, which makes detection and complete eradication difficult [25]. Similar to the literature, $C$. acnes was also the most common isolated microorganism in our cohort affecting almost half of the patients with shoulder PJI, followed by coagulase-negative staphylococci. Accordingly, low-virulent microorganisms represented almost $75 \%$ of all shoulder PJIs in patients with a normal CRP level.

Given the lack of strong evidence defining the clinical relevance of low-grade infections, new ICM definition criteria divided shoulder PJI into 4 subgroups, which allows for more homogenous groups with different characteristics of PJI and level of inflammation. In our study, patients with a definitive infection showed significantly higher serum CRP levels compared to patients with probable or possible infections. While mostly high-virulent microorganisms were evident in patients with definitive infection, such as Staphylococcus aureus or streptococci, interestingly four patients had an infection with $C$. acnes. One patient had a polymicrobial infection with C. acnes and Staphylococcus aureus, whereas the remaining three patients had a monomicrobial infection with $C$. acnes. These patients were classified as definitively infected due to the presence of a sinus tract or gross intra-articular pus. This difference in pathogenic potential and level of host inflammatory response might be caused by different phylotypes of $C$. acnes with varying virulence properties [26-28]. Boyle et al. were recently able to show that hemolytic strains of $C$. acnes exhibit enhanced pathogenicity to their host by eliciting a more prominent systemic inflammatory response and severe clinical course [26]. Furthermore, they reported a significantly higher median serum CRP level $(17.9 \mathrm{mg} / \mathrm{l})$ in the hemolytic $C$. acnes group compared to the nonhemolytic group $(3.5 \mathrm{mg} / \mathrm{l})$. Thus, CRP seems to be a more sensitive serum marker for inflammation than erythrocyte sedimentation rate and blood leucocyte count.
This study has limitations. First, we did not exclude patients with inflammatory diseases, which can alter our results. However, it was shown in the literature that there is no difference in the threshold of CRP level used to diagnose PJI in patients with or without an inflammatory disease [5]. Second, it was a retrospective analysis with lack of every available data for each patient. Third, despite using the last proposed definition criteria for shoulder PJI, some patients may be misdiagnosed as infected and vice versa.

In conclusion, serum CRP showed a low sensitivity and specificity for the diagnosis of shoulder PJI, even when applying cutoffs optimized using receiver-operating curve analysis. Low-virulent microorganisms and patients with probable and possible infections are associated with lower CRP levels compared to patients with definitive infection and infections caused by high-virulent microorganisms.

Funding Open Access funding enabled and organized by Projekt DEAL.

\section{Compliance with ethical standards}

Conflict of interest The authors declare that they have no conflict of interest.

Ethical approval The study protocol (Application number: EA4/040/14) was reviewed and approved by the institutional review board (Charite Universitätsmedizin).

Open Access This article is licensed under a Creative Commons Attribution 4.0 International License, which permits use, sharing, adaptation, distribution and reproduction in any medium or format, as long as you give appropriate credit to the original author(s) and the source, provide a link to the Creative Commons licence, and indicate if changes were made. The images or other third party material in this article are included in the article's Creative Commons licence, unless indicated otherwise in a credit line to the material. If material is not included in the article's Creative Commons licence and your intended use is not permitted by statutory regulation or exceeds the permitted use, you will need to obtain permission directly from the copyright holder. To view a copy of this licence, visit http://creativecommons.org/licenses/by/4.0/.

\section{References}

1. McGoldrick E, McElvany MD, Butler-Wu S, Pottinger PS, Matsen FA 3rd (2015) Substantial cultures of Propionibacterium can be found in apparently aseptic shoulders revised three years or more after the index arthroplasty. J Shoulder Elbow Surg 24:31-35. https://doi.org/10.1016/j.jse.2014.05.008

2. Koh CK, Marsh JP, Drinkovic D, Walker CG, Poon PC (2016) Propionibacterium acnes in primary shoulder arthroplasty: rates of colonization, patient risk factors, and efficacy of perioperative prophylaxis. J Shoulder Elbow Surg 25:846-852. https://doi. org/10.1016/j.jse.2015.09.033

3. Mahylis JM, Entezari V, Karichu J, Richter S, Derwin KA, Iannotti JP, Ricchetti ET (2018) Hemolytic strains of Propionibacterium 
acnes do not demonstrate greater pathogenicity in periprosthetic shoulder infections. J Shoulder Elbow Surg 27:1097-1104. https ://doi.org/10.1016/j.jse.2017.12.025

4. Parvizi J, Della Valle CJ (2010) AAOS Clinical Practice Guideline: diagnosis and treatment of periprosthetic joint infections of the hip and knee. J Am Acad Orthop Surg 18:771-772. https:// doi.org/10.5435/00124635-201012000-00007

5. Zmistowski B, Della Valle C, Bauer TW, Malizos KN, Alavi A, Bedair H, Booth RE, Choong P, Deirmengian C, Ehrlich GD, Gambir A, Huang R, Kissin Y, Kobayashi H, Kobayashi N, Krenn V, Drago L, Marston SB, Meermans G, Perez J, Ploegmakers JJ, Rosenberg A, Simpendorfer C, Thomas P, Tohtz S, Villafuerte JA, Wahl P, Wagenaar FC, Witzo E (2014) Diagnosis of periprosthetic joint infection. J Arthroplasty 29:77-83. https://doi.org/10.1016/j. arth.2013.09.040

6. Akgun D, Muller M, Perka C, Winkler T (2018) The serum level of C-reactive protein alone cannot be used for the diagnosis of prosthetic joint infections, especially in those caused by organisms of low virulence. Bone Joint J 100-B:1482-1486. https:// doi.org/10.1302/0301-620X.100B11.BJJ-2018-0514.R1

7. Akgun D, Burger J, Pumberger M, Putzier M (2019) C-reactive protein misdiagnoses delayed postoperative spinal implant infections in patients with low-virulent microorganisms. Eur Spine J. https://doi.org/10.1007/s00586-019-05889-3

8. Kheir MM, Tan TL, Shohat N, Foltz C, Parvizi J (2018) Routine diagnostic tests for periprosthetic joint infection demonstrate a high false-negative rate and are influenced by the infecting organism. J Bone Joint Surg Am 100:2057-2065. https://doi. org/10.2106/JBJS.17.01429

9. Fink B, Schlumberger M, Beyersdorff J, Schuster P (2020) C-reactive protein is not a screening tool for late periprosthetic joint infection. J Orthop Traumatol 21:2. https://doi.org/10.1186/s1019 5-020-0542-2

10. Dennison T, Alentorn-Geli E, Assenmacher AT, Sperling JW, Sanchez-Sotelo J, Cofield RH (2017) Management of acute or late hematogenous infection after shoulder arthroplasty with irrigation, debridement, and component retention. J Shoulder Elbow Surg 26:73-78. https://doi.org/10.1016/j.jse.2016.05.018

11. Dodson CC, Craig EV, Cordasco FA, Dines DM, Dines JS, Dicarlo E, Brause BD, Warren RF (2010) Propionibacterium acnes infection after shoulder arthroplasty: a diagnostic challenge. J Shoulder Elbow Surg 19:303-307. https://doi.org/10.1016/j.jse.2009.07.065

12. Grosso MJ, Sabesan VJ, Ho JC, Ricchetti ET, Iannotti JP (2012) Reinfection rates after 1-stage revision shoulder arthroplasty for patients with unexpected positive intraoperative cultures. J Shoulder Elbow Surg 21:754-758. https://doi.org/10.1016/j. jse.2011.08.052

13. Nelson GN, Davis DE, Namdari S (2016) Outcomes in the treatment of periprosthetic joint infection after shoulder arthroplasty: a systematic review. J Shoulder Elbow Surg 25:1337-1345. https ://doi.org/10.1016/j.jse.2015.11.064

14. Piper KE, Fernandez-Sampedro M, Steckelberg KE, Mandrekar JN, Karau MJ, Steckelberg JM, Berbari EF, Osmon DR, Hanssen AD, Lewallen DG, Cofield RH, Sperling JW, Sanchez-Sotelo J, Huddleston PM, Dekutoski MB, Yaszemski M, Currier B, Patel R (2010) C-reactive protein, erythrocyte sedimentation rate and orthopedic implant infection. PLoS ONE 5:e9358. https://doi. org/10.1371/journal.pone.0009358

15. Garrigues GE, Zmistowski B, Cooper AM, Green A, Group ICMS (2019) Proceedings from the 2018 International Consensus Meeting on Orthopedic Infections: the definition of periprosthetic shoulder infection. J Shoulder Elbow Surg 28:S8-S12. https:// doi.org/10.1016/j.jse.2019.04.034

16. Renz N, Feihl S, Cabric S, Trampuz A (2017) Performance of automated multiplex PCR using sonication fluid for diagnosis of periprosthetic joint infection: a prospective cohort. Infection 45:877-884. https://doi.org/10.1007/s15010-017-1073-5

17. Akgun D, Maziak N, Plachel F, Siegert P, Minkus M, Thiele K, Moroder P (2020) The role of implant sonication in the diagnosis of periprosthetic shoulder infection. J Shoulder Elbow Surg. https ://doi.org/10.1016/j.jse.2019.10.011

18. Shohat N, Bauer T, Buttaro M, Budhiparama N, Cashman J, Della Valle CJ, Drago L, Gehrke T, Marcelino Gomes LS, Goswami K, Hailer NP, Han SB, Higuera CA, Inaba Y, Jenny JY, KjaersgaardAndersen P, Lee M, Llinas A, Malizos K, Mont MA, Jones RM, Parvizi J, Peel T, Rivero-Boschert S, Segreti J, Soriano A, Sousa R, Spangehl M, Tan TL, Tikhilov R, Tuncay I, Winkler H, Witso E, Wouthuyzen-Bakker M, Young S, Zhang X, Zhou Y, Zimmerli W (2019) Hip and knee section, what is the definition of a periprosthetic joint infection (PJI) of the knee and the hip? Can the same criteria be used for both joints? Proceedings of international consensus on orthopedic infections. J Arthroplasty 34:S325-S327. https://doi.org/10.1016/j.arth.2018.09.045

19. Deirmengian CA, Citrano PA, Gulati S, Kazarian ER, Stave JW, Kardos KW (2016) The C-reactive protein may not detect infections caused by less-virulent organisms. J Arthroplasty 31:152155. https://doi.org/10.1016/j.arth.2016.01.060

20. McArthur BA, Abdel MP, Taunton MJ, Osmon DR, Hanssen AD (2015) Seronegative infections in hip and knee arthroplasty: periprosthetic infections with normal erythrocyte sedimentation rate and C-reactive protein level. Bone Joint J 97-B:939-944. https ://doi.org/10.1302/0301-620X.97B7.35500

21. Perez-Prieto D, Portillo ME, Puig-Verdie L, Alier A, Martinez S, Sorli L, Horcajada JP, Monllau JC (2017) C-reactive protein may misdiagnose prosthetic joint infections, particularly chronic and low-grade infections. Int Orthop 41:1315-1319. https://doi. org/10.1007/s00264-017-3430-5

22. Singh JA, Sperling JW, Schleck C, Harmsen WS, Cofield RH (2012) Periprosthetic infections after total shoulder arthroplasty: a 33-year perspective. J Shoulder Elbow Surg 21:1534-1541. https ://doi.org/10.1016/j.jse.2012.01.006

23. Hoiby N, Ciofu O, Johansen HK, Song ZJ, Moser C, Jensen PO, Molin S, Givskov M, Tolker-Nielsen T, Bjarnsholt T (2011) The clinical impact of bacterial biofilms. Int J Oral Sci 3:55-65. https ://doi.org/10.4248/IJOS11026

24. Zimmerli W, Sendi P (2017) Orthopaedic biofilm infections. APMIS 125:353-364. https://doi.org/10.1111/apm.12687

25. Mook WR, Garrigues GE (2014) Diagnosis and management of periprosthetic shoulder infections. J Bone Joint Surg Am 96:956965. https://doi.org/10.2106/JBJS.M.00402

26. Boyle KK, Nodzo SR, Wright TE, Crane JK, Duquin TR (2019) Hemolysis is a diagnostic adjuvant for Propionibacterium acnes orthopaedic shoulder infections. J Am Acad Orthop Surg 27:136144. https://doi.org/10.5435/JAAOS-D-17-00394

27. Nakatsuji T, Tang DC, Zhang L, Gallo RL, Huang CM (2011) Propionibacterium acnes CAMP factor and host acid sphingomyelinase contribute to bacterial virulence: potential targets for inflammatory acne treatment. PLoS ONE 6:e14797. https://doi. org/10.1371/journal.pone.0014797

28. Achermann Y, Goldstein EJ, Coenye T, Shirtliff ME (2014) Propionibacterium acnes: from commensal to opportunistic biofilmassociated implant pathogen. Clin Microbiol Rev 27:419-440. https://doi.org/10.1128/CMR.00092-13

Publisher's Note Springer Nature remains neutral with regard to jurisdictional claims in published maps and institutional affiliations. 